\title{
Measuring the frequency of "severe" accidental injury in childhood
}

\author{
S S Walsh, S N Jarvis
}

\begin{abstract}
Study Objective-The aim was to improve the epidemiological information concerning child accidental injuries which can be extracted from routine inpatient and coroners' inquest data.

Design-This was a retrospective study of coroners' inquest reports and inpatient case notes to undertake objective severity scaling and to extract basic data. This material was related to denominators from OPCS midyear population estimates, to 1981 census ward populations, and to sociodemographic data collected in a local census in 1986.

Setting-The study population was derived- from three north east health districts and their corresponding census enumeration districts.

Participants-A stratified sample of 500 children aged 0-16 years from among residents admitted to hospital with accidental injuries in 1986 was studied, together with all accidental injury child deaths between 1980 and 1986; $94 \%$ of the relevant case notes were localised and extracted.
\end{abstract}

Main results-When differentiated by injury severity there are major systematic differences in the basic epidemiology of child accidental injury by age and place of residence of victims as well as in the nature and causes of injuries sustained.

Conclusions-Injury severity scores can be used to define a "severity" threshold, within the spectrum of injuries, leading to hospital admission or death. By ensuring complete ascertainment this technique can provide a more accurate case definition than crude admission rates for estimating the frequency of injury in a population of children.

For most child illnesses, mortality is now such a rare event that epidemiological research must be based on morbidity data where the larger numbers can provide more timely and local information. Hospital inpatient data are the obvious choice for such morbidity information, first because they notionally contain the more "severe" cases, second because the quantity and quality of the information routinely held on each "case" is greater than at any other health service contact point, and third because such research can be undertaken at modest cost.

Hospital death or discharge rates are often used as a measure of the community frequency of illness and to represent the distributes of the disease in the community. However, these estimates suffer from the basic flaw of "selection bias"1 because the decision for a patient to attend a hospital and the decision of a doctor to admit a patient are both influenced by factors that are extraneous to the disease itself. ${ }^{2}$ Examples include ease of access to the hospital, bed supply, local admission policies, and social class of the patient. ${ }^{3-5}$ It is clear that for most diseases a large pool of unadmitted potential "cases" exists in the community at any particular time and that the probability of admission will increase with the "severity" of the condition.

Such selection biases present a number of problems when examining the frequency of the "disease" that is the greatest cause of mortality, acquired disability and morbidity in childhood, namely accidental injury. First, we must recognise that the term "accidental injury" covers a very wide spectrum of insults ranging from minor lacerations of skin to massive crush injuries of the brain, and that the community frequency reduces dramatically as we move up the severity spectrum. This gives rise to a large "iceberg" of injuries in the community stratified by severity ${ }^{6}$ of which we see only the tip in hospital. Second, over the last 20 years there have been important changes in the supply and use of paediatric beds ${ }^{7}$ as well as specific modifications in the management of injured children. For instance, hospital admission rates for childhood accidental injury rose during the 1960 s and early 1970 s, since when they have declined. ${ }^{8}$ Does this reflect true changes in accident rates in the community or merely an artefact of selection bias? It seems likely that the major influences on paediatric admission rates for accidental injury are not the numbers of injuries in the community but rather bed supply and clinical policy biases affecting a large potential pool of minor injuries.

One methodology that might overcome these problems is severity grading of illness. Much used in America in the context of "case mix" for medical audit and payment (eg, diagnosis related groups, severity of illness, and disease staging) ${ }^{9}$ such a severity grading can also have epidemiological uses. An objective severity scale that would identify a threshold of severity beyond which we could be reasonably assured that all such cases occurring in the community would inevitably have presented and have been admitted to hospital, would allow the use of hospital inpatient data to achieve the goal of providing a complete and unbiased set of well defined cases.

It is proposed in this paper that the addition of a "severity" grading to routine hospital diagnostic data for injuries can reveal not only whether trends in rates of hospital admissions reflect the 
true underlying community trends, but also will allow attention to be focused on the determinants of injury at varying levels of severity, thereby helping to set priorities for primary prevention. To support these contentions we present results from a retrospective study which used an objective severity scale to show how hospital inpatient data and coroners' inquest data concerning children injured in accidents can be enhanced by the addition of severity grading.

\section{Methods}

The study population was children under the age of 16 years resident in part of the Northern Region-Newcastle upon Tyne, North Tyneside, and Northumberland-with a total childhood population of 153000 .

The regional information systems section of the Northern Regional Health Authority provided a sample of 500 children admitted to hospital during 1986 with accidental injury from hospital activity analysis data files. These were drawn from the total number of 1775 eligible cases, stratified by age group, sex, area of residence, and diagnostic group, and randomly selected from within strata. Each case was identified by their hospital record number, the relevant hospital, the dates of admission and discharge, and the consultant in charge.

The information section also supplied a list of all the 118 child deaths registered with an underlying cause of "injury and poisoning" between 1980 and 1986 from the Office of Population Censuses and Surveys (OPCS) "death tape". From this list, the death certificate and thence the name of the child, the date of the inquest, and the identity of the responsible coroner were obtained. With the permission from the appropriate consultant/coroner we examined the relevant case notes/inquest files. Epidemiological and injury severity data were extracted onto a proforma for later transfer to an anonymous file on the university computer mainframe.

For severity grading we used the Abbreviated Injury Scale (AIS). ${ }^{10}$ This has the advantage of scaling directly the injury itself or the clinical status of the patient separate from any extrinsic influences. In particular the AIS avoids the use of treatment derived data as is common in other severity categorisations (eg, diagnosis related groups)

The AIS is a non-linear six point scale based mainly on a "threat to life" criterion and applied to six different body regions (eg, head, abdomen, skeletal system). An overall score (ranging from 1-75) can be calculated for the patient by summing the squares of the three highest AIS part scores to form the injury severity score (ISS). ${ }^{11}$ This severity scoring system has been applied to both adult and paediatric populations and found to be reliable and valid. ${ }^{12}$ The single disadvantage is that the AIS does not code non-traumatic injury (eg, poisoning, asphyxia).

We attempted to estimate the point along the injury severity spectrum that would entail certain hospital admission (or death) for all such cases occurring in the community. For traumatic cases an ISS of $\geqslant 9$ was judged sufficiently "severe" for this purpose. Examples of this degree of severity include fractured femur, fractured ribs with haemothorax, fractured base of skull, $30 \%$ to $40 \%$ burns, etc. In addition, we classified an ISS of $\geqslant 4$ as representing a group of children with at least a "moderate" injury. Examples include undisplaced long bone fractures, head injury with loss of consciousness.

For non-traumatic cases the following cases were judged to be equivalent to the "severe" category of traumatic injury:

$$
\begin{aligned}
& \text { Poisoning - Proven ingestion of a lethal } \\
& \text { substance } \\
& \text { - History of ingestion with a } \\
& \text { revised trauma score } \\
& \text { (triage) }<10 \\
& \text { - Lodged in airway } \\
& \text { - Perforated viscus }
\end{aligned}
$$

The revised trauma score (triage) (T-RTS) $)^{13}$ is a linear 12 point scale based on the patient's cardiovascular, respiratory, and central nervous systems. It also is reliable and valid when applied to children. ${ }^{10}$

Denominator data were obtained by using the mid-year population estimates for the three district health authorities as supplied by the OPCS. For inpatient statistics we used the 1986 mid-year estimate, while for deaths the mean mid-year population for the years 1980-86 was determined.

For more detailed small area analysis the 1981 census data were obtained for the appropriate electoral ward populations. A household mini census conducted in Newcastle upon Tyne in 1986 provided valuable data to verify the current ward level social characteristics. To assign the cases to the correct geographical area the post code of each injured child was translated into an electoral district and thence to a ward by means of its grid reference using a computer software package supplied by the University Geography Department.

\section{Results}

From the 500 hospital admissions identified, a total of $482(96.4 \%)$ case records were located. Of these $\mathbf{4 1 5}$ satisfied the criteria for entry into the study. The reasons for non-elegibility included: intentional poisoning 29 ; miscoding by hospital activity analysis 19; non-accidental injury 9 ; assault 5; old injury 4 .

Of the 118 deaths identified, 95 were analysed. Two coroner's inquest files could not be located. The remainder were determined ineligible because the injury was not a consequence of an "accident" (eg, non-accidental injury, intentional poisoning, inattention at birth) or concerned non-resident children. Of the children admitted to hospital only one child died (and only appears in the data relating to deaths). 
All 415 hospital cases were given a severity grading and for 132 of these cases the injuries were judged "severe" by the criteria outlined in "Methods". For deaths all cases were as a consequence of "severe" injuries by the same criteria.

Annual rates (per million population) are presented first, for "all" injuries (ie, the picture presented by unrefined hospital data); second, for "severe" injuries (in some instances supplemented by a "moderate" group); and third, for "deaths". It is important to note that the categories "all", "moderate", and "severe" injuries are representing cumulative rather than distinct rates (eg, "moderate" includes any injury admission at or beyond an ISS score of 4 and therefore includes "severe" injuries). However death rates are depicted separately because they come from a different sampling frame and base denominator population.

Log scales are used in order to represent death and admission rates on the same figure.

Figures 1 and 2 show the frequency of injury classified by age first for the whole sample and next for traumatic injuries only. In fig 1 it is

Figure 1 Rates of accidental injury by age subdivided by severity

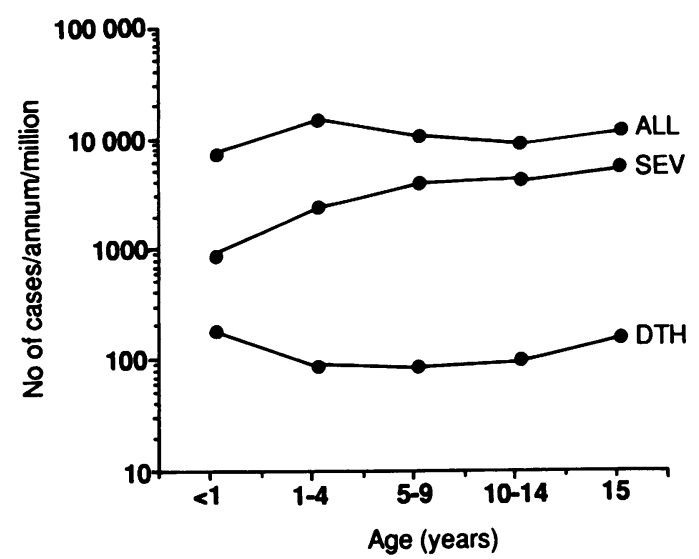

noticeable that the age group 1-4 years has the highest rate of hospital admission (ie, "all" injuries). For "severe" injuries, however, there is a more linear relationship between injury frequency and age and it will be recalled that the underlying proposition is that this represents a more accurate estimate of community frequency. When examining deaths a further different picture emerges. Infants have the highest rate, but thereafter the graph is similar to severe injuries.

The causes of these accidental deaths in infancy are largely "non-traumatic" in nature (eg, suffocations, aspirations, etc) but interestingly these are not proportionately represented in hospital data. Therefore, we examine in fig 2 the separate relationship between "traumatic" injury (eg, fractures, head injury, etc) and age. This had the additional advantage that we can define a group of children with "moderate" injury (eg, ISS $\geqslant 4$ ). Again the increased frequency for the 1-4 years age group for "all" injury is noted. However as we progress up the severity spectrum the three lines for moderate, severe, and fatal injury are virtually parallel.

We suggest an appropriate interpretation of fig 2 is that there is a selection bias towards admitting younger children, principally affecting those with mild injury (ie, less than moderate) and that this

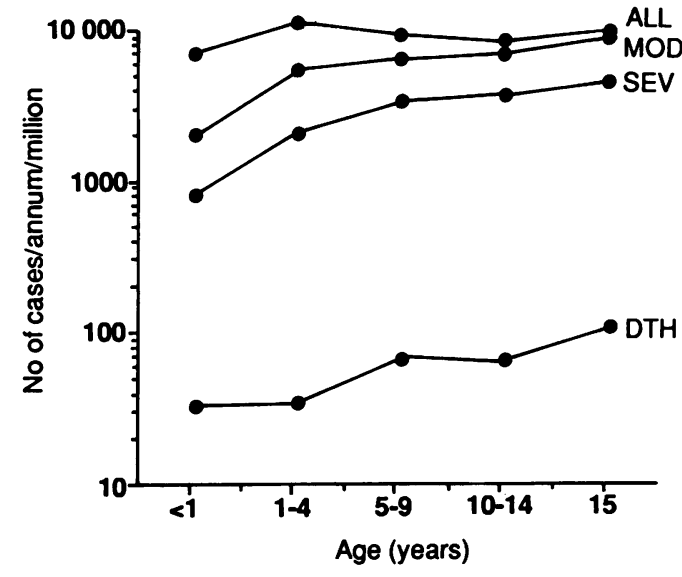

Figure 2 Rates of accidental injury by age subdivided by severity (excluding "non-traumatic" injuries)

overall admission rate (top line in fig 2) does not represent the true community picture. On the contrary it appears that the true frequency of traumatic injury in the community increases progressively with age and has a uniform distribution by severity. Therefore not only does the "severe" group indicate the true frequency for that end of the severity spectrum, it also gives us some information on the community incidence for all injuries and highlights some of the systematic errors encountered in the interpretation of crude hospital admission data.

Figure 3 shows the distribution of injury by type. This demonstrates the importance of fractures and dislocations not just in terms of admissions but also because a high proportion of them are "severe". This contrasts with head injuries and poisonings which, although major causes of admission, are not correspondingly evident in severe injuries.

It must also be emphasised that the purpose of this exercise is not to suggest that individual doctors are wrong to admit patients with certain mild injuries for observation, but rather to demonstrate the potential for overrepresenting head injury and poisoning as a community problem. Indeed it is clear that head injury is a major contributor to the small numbers of deaths and for this reason "observation" of apparently mild cases may be necessary.

Figure 4 shows the distribution by cause. The category "road traffic accident" best demonstrates the utility of a severity classification From overall admission rates it would appear that road traffic accidents make only a small contribution to hospital admissions. However, if we concentrate on severe injury and death the contribution of road traffic accidents as causes of injury increases dramatically. The natural corollary of this would also be evident if we examined the place of injury (not shown) Although the home is the site of accident in the majority of cases admitted to hospital, especially for the younger children, the environment outside the home, and in particular, exposure to road traffic, is probably the most important risk factor for the more serious injuries.

Figures 5-7 present an analysis of accidents on a geographical basis by using the postcodes of the affected Newcastle and North Tyneside children. 


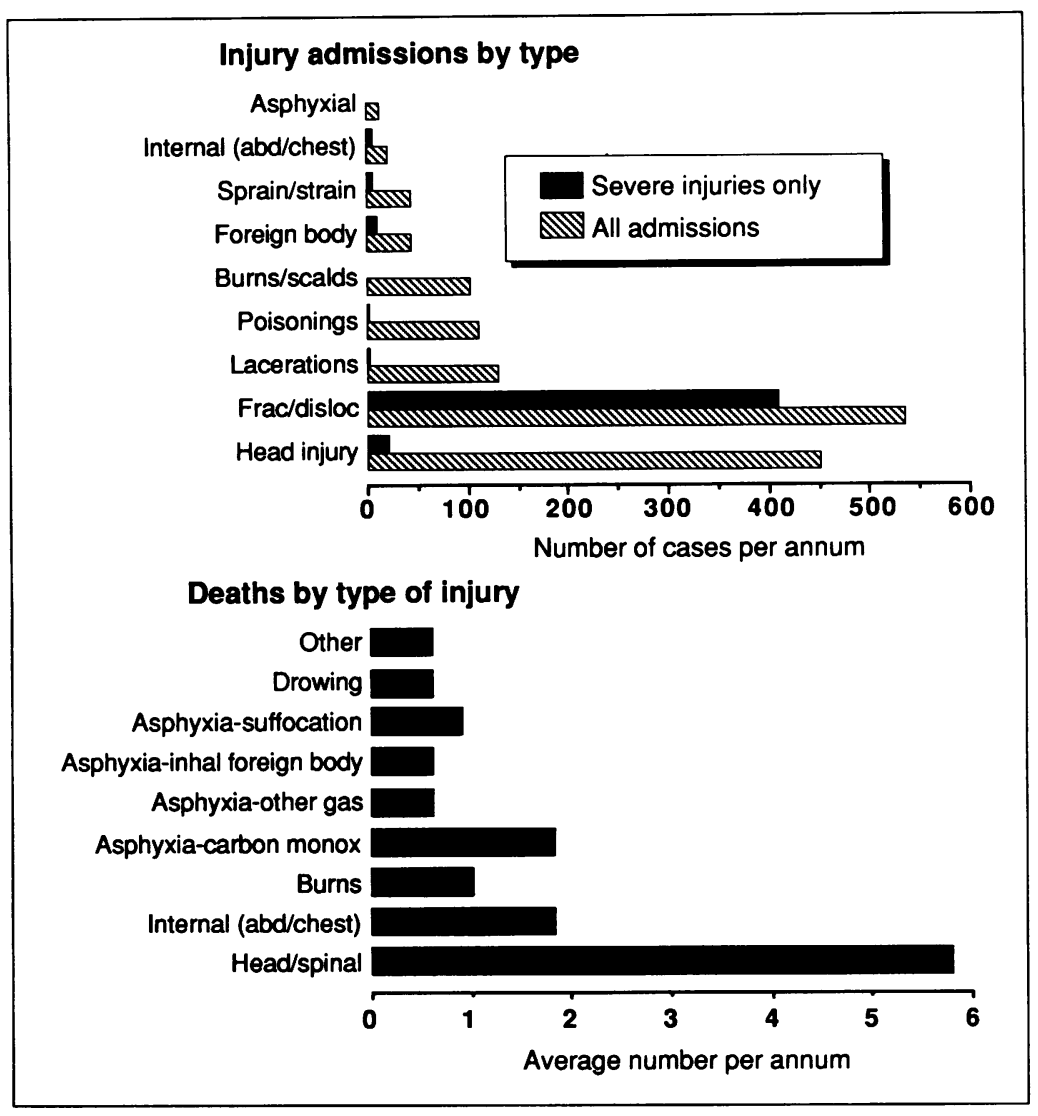

Figure 3 Type of injury for (a) hospital admissions and (b) deaths

Northumberland children are excluded because of the difficulties in map interpretation of large rural areas. The 49 urban electoral wards are divided into quartiles on the basis of rates for each category of injury.

Looking at the three maps together, it can be seen that the question of where in the city above average rates are concentrated must be tempered by what level of severity classification we accept. Those areas of the city that have the highest "all injury" rates are not necessarily the same areas where severe injury and death are occurring. Indeed, there are only poor correlations between the rates of injury, across the 49 wards, for the three levels of severity (non-severe injury, severe injury, and deaths; table I). This has obvious implications for the planning of an accident prevention programme. It emphasises that the correct targeting of high risk areas ought to encompass some data beyond routine hospital

Table I Rank correlation of injury rates for the 49 census wards at three levels of severity

\begin{tabular}{lll}
\hline & $\begin{array}{l}\text { Spearman's } \\
\text { correlation } \\
\text { coefficient }\end{array}$ & p Value \\
\hline Non-severe $\star$ /severe & 0.08 & 0.56 \\
Non-severe/death & 0.13 & 0.37 \\
Severe/death & 0.12 & 0.41 \\
\hline ॠNon-severe = "all" injuries minus "severe" injuries, to avoid \\
autocorrelation
\end{tabular}

statistics. It is also clear that the ecological correlates of the injury rate will be dependent on the level of "severity" of injury that is used. If, say, the unemployment rate is found to be strongly associated with the "all" injury rate in the same wards, does this represent a "true" association or an artefact due to "selection bias" towards admitting children from those wards marked by high unemployment?

For the purposes of this paper, we have confined such an "ecological" study to a preliminary analysis of Newcastle upon Tyne wards because here a 1986 household survey provides more up to date sociodemographic data. ${ }^{14} \mathrm{We}$ were able to use information from this to obtain accurate denominators and to confirm that the Ward ranking by deprivation as performed by Townsend et $a l^{15}$ in 1981 had remained the same in 1986. In fig 8 and table II the 26 electoral wards of the city are divided into quartiles defined by Townsend deprivation rank. In the figure these quartiles are shown on the " $x$ "

Table II Injury rates by ward groupings (per 100000 ). Figures in brackets represent actual number of cases

\begin{tabular}{lccc}
\hline Wards & \multicolumn{1}{l}{ All } & Severe & \multicolumn{1}{c}{ Death } \\
\hline A & $7564 \cdot 0(25)$ & $1512 \cdot 0(5)$ & $24 \cdot 4(2)$ \\
B & $12300 \cdot 0(40)$ & $3693 \cdot 0(12)$ & $50.5(4)$ \\
C & $15000 \cdot 0(51)$ & $4925 \cdot 0(16)$ & $123 \cdot 0(10)$ \\
D & $18000 \cdot 0(64)$ & $5381 \cdot 0(19)$ & $183 \cdot 0(16)$ \\
Relative rate (D/A) & $2 \cdot 4$ & $3 \cdot 6$ & $7 \cdot 5$
\end{tabular}

axis, " $A$ " representing the most affluent and " $D$ " the most deprived wards. Areas of social deprivation have the highest injury rate with a relative rate (RR) of deprived against affluent quartiles for "all" cases of 2.4 (95\% CI 1.5-3.8, $\mathrm{p}<0.05$ ); with a progressive increase in this differential for severe cases, RR $3.6(95 \%$ CI $1.3-9.5, \mathrm{p}<0.05)$; and deaths, RR $7.5(95 \% \mathrm{CI}$ $1.8-32.6 \mathrm{p}<0.05)$. This suggests not only that the rate of accidental injury is increased in poorer areas but also that the distribution of injuries is disproportionately skewed to the more severe end of the severity spectrum in these same areas.

An alternative interpretation of the high relative rate for deaths could be that children from deprived areas die at lower levels of injury severity than children from affluent wards. We have therefore analysed this association in more detail by using information on individual social class (by occupation of head of household) which was available for 71 of the 95 deaths. We compared the ranking of severity scores resulting in death and found no significant difference between the manual and non-manual social class groups (Mann Whitney $U$ test, $p=0 \cdot 4$ ). This implies that poorer children are not more likely to die at a given severity score than more affluent children. Ideally, this association should be studied using a case fatality method (ie, the proportions of children who die at different severity intervals across social classes). Unfortunately individual social class was insufficiently recorded amongst hospital admissions.

The results presented have focused on those variables where the distribution of injury rates change systematically at different points on the severity spectrum. However, we have also found that for certain variables, namely, hour, day, and month of accident, and for sex of child, the injury rates do not differ by severity classification. This would seem to imply that for these variables routine hospital statistics do represent the community distribution. 


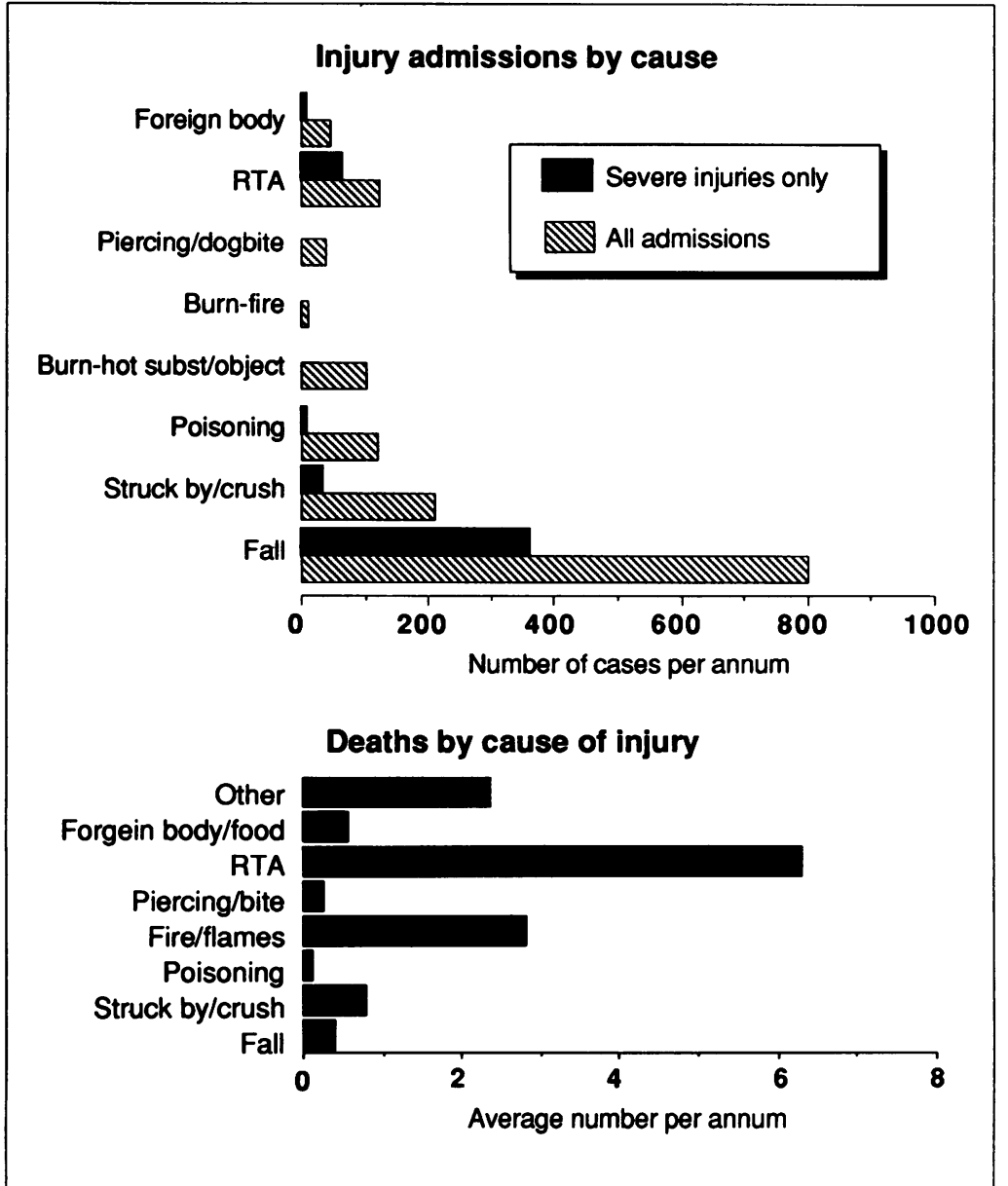

Figure 4 Cause of injury for (a) hospital admissions and (b) deaths

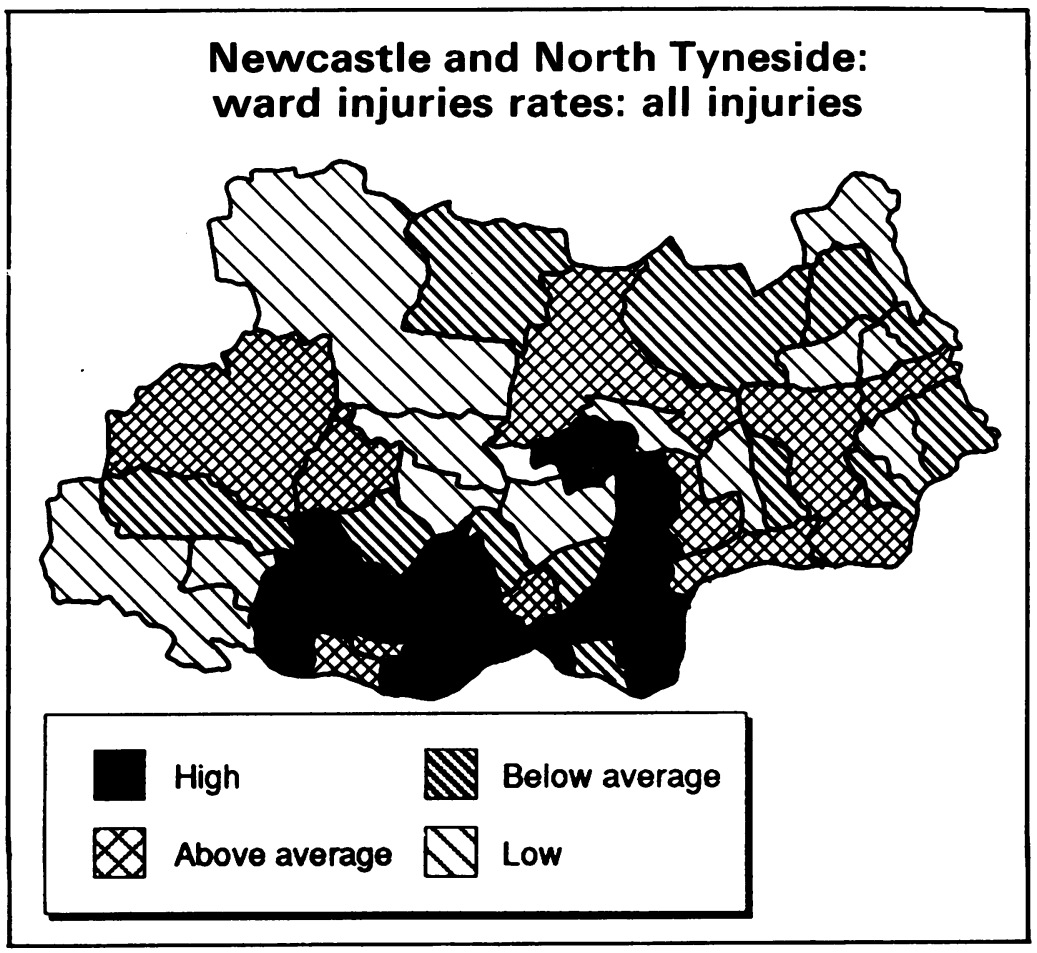

Figure 5 Injury admission rates for the 49 census wards of Newcastle upon Tyne/ North Tyneside

Discussion

Child accident epidemiology is increasingly concerned with morbidity data and the use of routine hospital inpatient statistics. As outlined by Anderson ${ }^{16}$ such hospital statistics serve two purposes, first, administrative (use of hospital services and associated costs), and second, epidemiological (guide to morbidity occurrence in a community). The latter purpose, however, cannot be achieved until we can avoid the selection biases that interfere with the direct interpretation of admission rates as representative of community frequency.

It seems almost certain that such biases will principally affect the "milder" cases of any condition likely to lead to hospital admission and that therefore some form of "severity" classification will be necessary. Essentially there are two candidates for such a severity classification-indirect and direct indices.

Indirect indices (eg, operative intervention, length of stay, etc) are largely treatment based, and although relatively easy and cheap to collect are themselves susceptible to selection bias, which only serves to further confound and confuse an already entangled situation. In addition, they also prevent any investigation of the effect of treatment in improving patient outcome.

Direct indices such as the AIS, ISS, and trauma score used here have largely been used as triage tools, in clinical assessment, and for calculating "case mix" for prepaid American trauma centres. However such direct severity indices also lend themselves to epidemiological use by identifying an unbiased set of patients with severe injury. We have used this methodology to identify a group of children with "severe" injuries who we believe will inevitably present and be admitted to hospital regardless of extrinsic bias.

Much has been published on childhood accidental injury. Some workers have concentrated on basic descriptive epidemiology, 1718 others have performed analytical studies, ${ }^{1920}$ and yet others have described preventive studies. ${ }^{2122}$ Our basic knowledge and understanding of accidental injury have come from these studies. However, the great majority of such studies have relied on a "case definition" based on the arrival of injured children at a health service point for treatment. The importance of "case definition" in determining the results obtained from accident research has been alluded to by Brown and co-workers using data from the Child Health and Education Study. ${ }^{23}$ They defined an accident as one "considered severe enough to "warrant medical attention" " and recognised that "seeking medical attention and the decision to admit to hospital are influenced by a variety of factors other than the injury itself'. The study examined the association of eight social and environmental factors with childhood accidents (using three separate measures of accidental injury: one or more accidents, two or more accidents, and hospital admission) and concluded that "variation in case selection can lead to different conclusions about the risk factors associated with childhood accidents". Interestingly the authors also stated that "an injury severity rating applicable to children" was required to assist in removing bias from accident research.

Other studies that have tried to address the notional concept of severity have relied heavily on treatment based criteria (eg, hospital admission, 


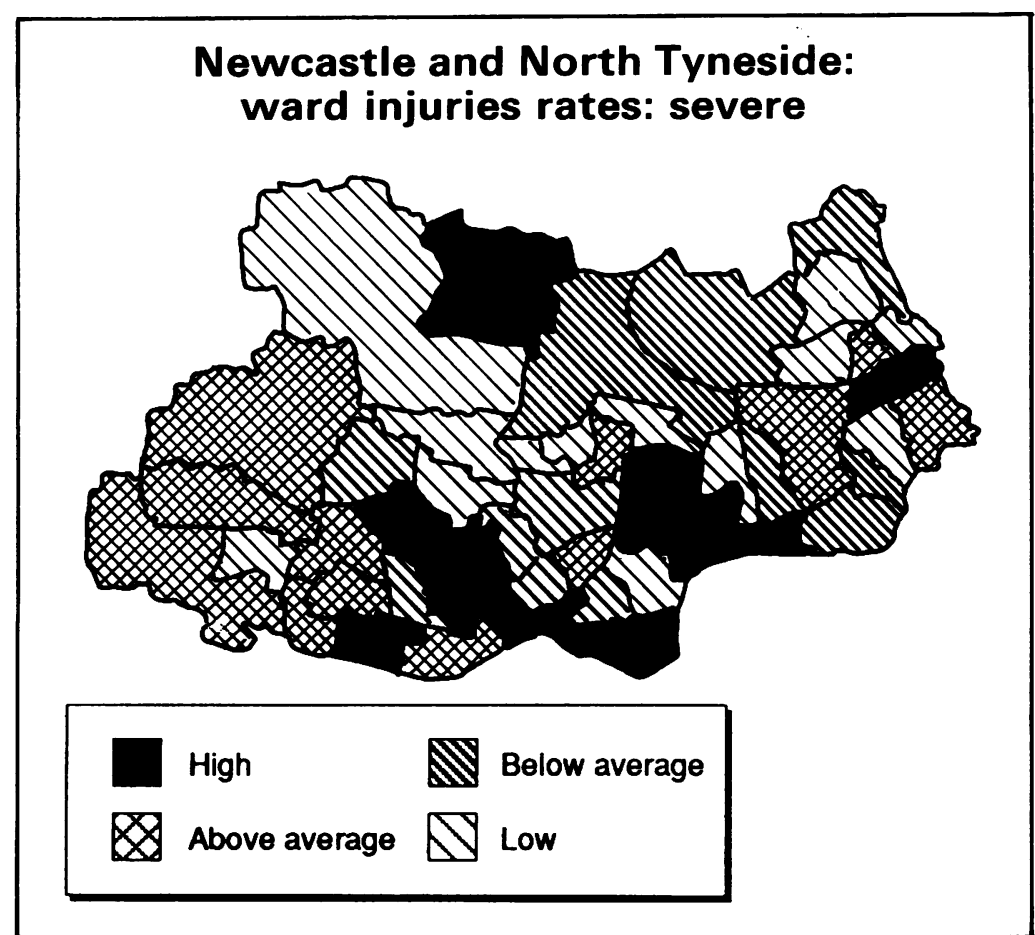

Figure 6 Severe injury admissions only for the 49 census wards of Newcastle upon Tyne/North Tyneside

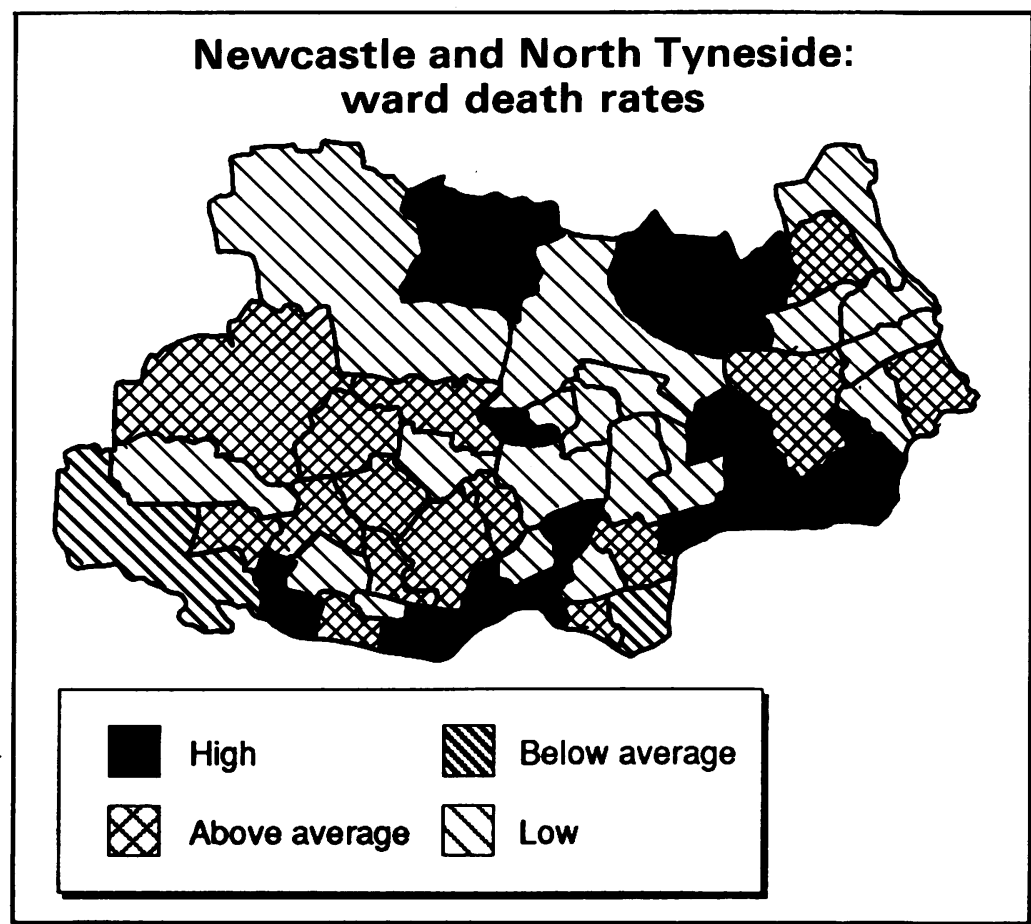

Figure 7 Injury deaths for the 49 census wards of Newcastle upon Tyne/North Tyneside

length of stay, number of attendances at outpatients). ${ }^{2425}$

Using a "direct" injury severity classification on paediatric inpatient data we have shown that we can not only illustrate the "true" community frequency at a particular level of injury severity but may also provide a community profile for the total spectrum of injury. Meanwhile in our examination of injury mortality (which almost certainly represents the true community rate) it is important to remember that death is an outcome and not an independent measure of severity.
Children can die from mild injuries and thus the classification of deaths by severity is as important as for morbidity data.

Beyond the demonstration of the feasibility of such severity coding of accidental injuries there are several other interesting findings from our study. There appears to be a relative excess of deaths in non-traumatic injury among infants (ie, under age 1 year). This raises the possibility that these injuries (eg, suffocation, drowning, etc) behave in an "all or none" fashion (ie, the infant either dies or recovers without ever requiring hospital admission). It would seem that the only type of intervention that could operate successfully here is primary prevention.

Preschool children (ie, age 1-4 years) have been the focus of much research on accidental injury on the basis of their high admission rates to hospital. $^{2526}$ However, on the evidence of this study, this is not a true reflection of a community problem. On the contrary we would suggest that more prevention resources need to be focused on the older age groups.

The similarity in rates of injury at different levels of severity by sex is interesting. Although boys have an overall rate twice that of girls in a variety of studies, ${ }^{27}$ it has never been fully explained.

The importance of falls as a major cause of injury in childhood has been documented by several researchers previously, both in terms of attendance at accident and emergency departments and admission to hospital. ${ }^{28}$ The fact that we found them to be the leading cause of "severe" injuries should lend even further impetus to the need for even greater public awareness of the problem and further preventative research.

Geographical techniques have a particular importance in the study of accidental injury because the host/environment interaction is a key element in the aetiology. Our analysis of ward rates emphasises the point that area based analysis should take some account of "severity" of disease if it is to be valid. It is interesting that a study by Constantinides and Walker ${ }^{25}$ in London, based on accident and emergency department attendances, found no differences in the geographical distributions by ward of mild, moderate, and severe injury, using a treatment based severity

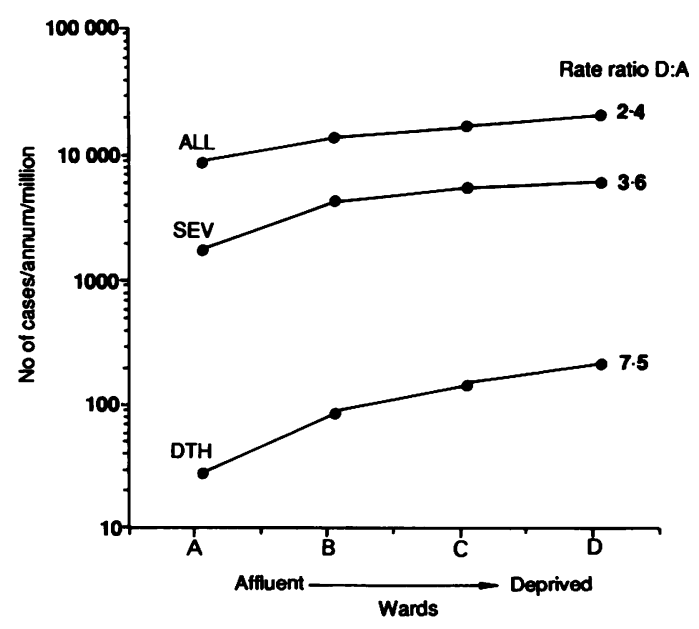

Figure 8 Injury frequency by sociodemographic grouping for all accident types subdivided by injury severity 
criterion. This contrasts sharply with our findings, using a more direct severity index, which show no significant correlation between the rates of three categories of injury admissions across wards (cf table I). Further ecological analysis of this material to address the issue of social deprivation and accidental injury is being undertaken and will be reported separately.

We conclude that the epidemiologist wishing to describe the frequency of illness, the community distributes, and the targeting, institution, and evaluation of preventative policies should obtain statistics for all hospital admissions, severe cases and deaths.

The study was funded by the Research Committee of the Northern Regional Health Authority. We acknowledge the help of Sheila Barton (Centre for Urban and Regional Development Studies, University of Newcastle upon Tyne) in the preparation of the maps used in this paper. SSW is a Wellcome Trust Training Fellow in Clinical Epidemiology.

1 Rothman K. Modern epidemiology. Boston: Little, Brown and Co, 1986: 83-4.

and $\mathrm{Co}, 1986.83-4$. 1980s. London: Health Education Council, 1987: 46-50. 3 Milner P, Nicholl J, Williams B. Variation in demand for accident and emergency departments in England from 1974-85. F Epidemiol Community Health 1988; 42: 274-8. 4 Earthrowl B, Stacey M. Social
Soc Sci Med 1977; 11: 83-8.

Soc Sci Med 1977; 11: 83-8.
5 Wadsworth M, Morris S. Assessing chances of hospital 5 Wadsworth M, Morris S. Assessing chances of hospital admission.

6 Guyer B, Gallagher S. An approach to the epidemiology of childhood injuries. Pediatr Clin North Am 1985; 32: 5-15. 7 Hill A. Trends in paediatric medical admission. BMF 1989, 298: 1479-83.
8 Constanides P. The management response to childhood accidents. London: Kings Fund Centre Report, 1987; 2-3. 9 Cretin S, Worthman L. Alternative systems for case mix classification in health care financing. Washington DC: US Dept of Health and Human Services Report, 1986.

10 American Association for Automotive Medicine. The abbreviated injury scale. Illinois, USA, 1985.

11 Baker S, O'Neill B, Haddon W, et al. The Injury Severity Score. F Trauma 1974; 14: 187-96.

12 Wesson D, Spence L. Injury scoring systems in childhood. Can $\mathcal{F}$ Surg 1987; 30: 398-400.

13 Champion H, Sacco W, Copes W. A revision of the Trauma Score. 7 Trauma 1989; 29: 623-9.

14 Newcastle upon Tyne "City Profiles". City of Newcastle upon Tyne, 1988 .

15 Townsend $\mathrm{P}$, Phillimore $\mathrm{P}$, Beattie A. Health and deprivation: inequality and the North. London: Croom Helm, 1987 .

16 Anderson $\mathrm{H}$. The epidmiological value of hospital diagnostic Anders. In: Bennett A, ed. Recent advances in community data. In: Bennett A, ed. Recent advances in community medicine 1. London: Churchill Livingstone, 1978: 175-93
17 Gallagher S, Finison $\mathrm{K}$, Guyer $\mathrm{B}$. The incidence of injuries Gallagher S, Finison $\mathrm{K}$, Guyer $\mathrm{B}$. The ind adolescents. $A m$ among 87,000 Massachusetts child

F Public Health 1984; 74: 1340-7.
18 Sibert J, Maddocks G, Brown B. Childhood accidents-an endemic of epidemic proportions. Arch Dis Child 1981; 56 225-37.

19 Sibert J. Stress in families of children who have ingested poisons. $B M \mathcal{F}$ 1975; ii: 87-9.

20 Alwash R, McCarthy M. Accidents in the home amon children under five: ethnic differences or socia disadvantage? $B M \mathcal{f}$ 1988; 296: 1450-3.

21 Gallagher S, Hunter P, Guyer B. A home injury prevention program for children. Pediatr North Clin Am 1985; 32 95-113.

22 Spergil C, Lindman F. Children can't fly: a program to prevent childhood morbidity and mortality from window falls. Am $¥$ Public Health 1977; 67: 1143-7.

23 Stewart-Brown S, Peters T, Golding J, et al. Case definition in childhood accidents. Int 7 Epidemiol 1986; 15: 352-9.

Troop $P$. Accidents to children. An analysis of inpatient admissions. Public Health 1986; 100: 278-85.

admissions. Public Health 1986 , 100 . 278 - 85 . London borough. North East Thames Regional Health London borough. North

26 Meyer R, Roelofs H, Bluestone J, et al. Accidental injury to the preschool child. $\mathcal{F}$ Pediatr 1963; 63: 95-105.

27 Rivara F, Bergman A, Logerfo J, et al. Epidemiology of Rivara F, Bergman A, Logerfo J, et al. Epidemiology of childhood injuries: sex diffe

28 Meller J, Shermeta D. Falls in urban children. Am f Dis Child 1987; 141: 1271-5. 